Suzanne George, Qian Wang, James E. Butrynski, Jeffrey A. Morgan, Andrew J. Wagner, Jeffrey T. Yap, Annick D. Van den Abbeele, Judith B. Manola, Sarah M. Solomon, and George D. Demetri, Dana-Farber Cancer Institute; Edwin Choy, Massachusetts General Hospital; Meijun Zhu and Jonathan A. Fletcher, Brigham and Women's Hospital; Suzanne George, James E. Butrynski, Jeffrey A. Morgan, Andrew J. Wagner, Annick D. Van den Abbeele, Edwin Choy, Jonathan A. Fletcher, and George D. Demetri, Harvard Medical School, Boston, MA; Michael C. Heinrich and Christopher L. Corless, Oregon Health Sciences University, Portland, OR; William D. Tap, University of California at Los Angeles, Los Angeles, CA; and Margaret von Mehren, Fox Chase Cancer Center, Philadelphia, PA

Submitted October 13, 2011; accepted March 6, 2012; published online ahead of print at www.jco.org on May 21, 2012.

Supported in part by Bayer HealthCare Pharmaceuticals, which provided funding and study drug, and by National Cancer Institute (NCl) GI Specialized Programs of Research Excellence Grant No. 1P50CA127003 to Dana-Farber/Harvard Cancer Center (DFHCC; J.A.F., G.D.D.), a Merit Review grant from the Department of Veterans Affairs (M.C.H.), NCl Grant No. CA06927 (M.V.), the Virginia and Daniel K. Ludwig Trust for Cancer Research and the Ludwig Center at DFHCC (J.A.F., G.D.D.) and the Life Raft Group and the GI Stroma Tumor Cancer Research Fund (M.C.H. C.L.C., J.A.F.).

Authors' disclosures of potential conflicts of interest and author contributions are found at the end of this article.

Clinical Trials repository link available on JCO.org.

Corresponding author: Suzanne George, MD, Center for Sarcoma and Bone Oncology, Dana-Farber Cancer Institute D1212, 450 Brookline Ave, Boston, MA; e-mail: suzanne_george@dfci.harvard.edu.

C 2012 by American Society of Clinical Oncology

0732-183X/12/3019-2401/\$20.00

DOI: 10.1200/JCO 201139.9394

\title{
Efficacy and Safety of Regorafenib in Patients With Metastatic and/or Unresectable GI Stromal Tumor After Failure of Imatinib and Sunitinib: A Multicenter Phase II Trial
}

Suzanne George, Qian Wang, Michael C. Heinrich, Christopher L. Corless, Meijun Zhu, James E. Butrynski, Jeffrey A. Morgan, Andrew J. Wagner, Edwin Choy, William D. Tap, Jeffrey T. Yap, Annick D. Van den Abbeele, Judith B. Manola, Sarah M. Solomon, Jonathan A. Fletcher, Margaret von Mehren, and George D. Demetri

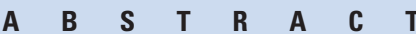

\section{Purpose}

Metastatic GI stromal tumor (GIST) is a life-threatening disease with no therapy of proven efficacy after failure of imatinib and sunitinib. Regorafenib is a structurally unique inhibitor of multiple cancer-associated kinases, including KIT and platelet-derived growth factor receptor (PDGFR), with broad-spectrum anticancer activity in preclinical and early-phase trials. Because KIT and PDGFR- $\alpha$ remain drivers of GIST after resistance to imatinib and sunitinib, we performed a multicenter single-stage phase II trial of regorafenib in patients with advanced GIST after failure of at least imatinib and sunitinib.

\section{Patients and Methods}

Patients received regorafenib orally, $160 \mathrm{mg}$ daily, on days 1 to 21 of a 28-day cycle. Disease assessment was performed every two cycles per RECIST 1.1. Primary end point was clinical benefit rate (CBR), defined as objective responses (ie, complete or partial response [PR] as well as stable disease $[S D] \geq 16$ weeks). Serial tumor biopsies were obtained from consenting patients whenever possible.

\section{Results}

From February to December 2010, 34 patients were enrolled at four US centers. As of July 28, 2011, 33 patients had received at least two cycles of regorafenib (range, two to 17 cycles). CBR was $79 \%(95 \% \mathrm{Cl}, 61 \%$ to $91 \%)$. Four patients achieved PR, and 22 exhibited SD $\geq 16$ weeks. Median progression-free survival was 10.0 months. The most common grade 3 toxicities were hypertension and hand-foot-skin reaction.

\section{Conclusion}

Regorafenib has significant activity in patients with advanced GIST after failure of both imatinib and sunitinib. A phase III trial of regorafenib versus placebo is ongoing to define more fully the safety and efficacy of regorafenib in this setting.

\section{J Clin Oncol 30:2401-2407. (C) 2012 by American Society of Clinical Oncology}

\section{INTRODUCTION}

Treatment of GI stromal tumor (GIST) was revolutionized after the identification of activating mutations in the KIT and PDGFR- $\alpha$ genes encoding structurally aberrant receptor tyrosine kinases, which serve as primary drivers of oncogenic signaling in this disease, and the development of potent inhibitors of these receptor tyrosine kinases. ${ }^{1,2}$ Despite dramatic responses and high rates of disease control with imatinib as first-line therapy for advanced GIST as well as disease control rates of $60 \%$ with median progression-free survival (PFS) of 27 weeks in the second-line setting with sunitinib, development of tyrosine kinase inhibitor (TKI) resistance eventually occurs in nearly all patients with advanced GIST. ${ }^{3-6}$ There is currently no third-line therapy approved by any regulatory authority in TKI-refractory metastatic GIST.

Regorafenib (BAY 73-4506) is a structurally distinct, orally available multikinase inhibitor with activity against multiple targets, including KIT, platelet-derived growth factor receptor (PDGFR), vascular endothelial growth factor receptor 1, R2, R3, TIE2, RET, fibroblast growth factor receptor 1, RAF, and p38 mitogen-activated protein kinase 
(MAPK). Regorafenib has shown potent antitumor activity in a variety of preclinical models, including inhibition of growth of GIST cell lines. ${ }^{7}$ Phase I trials have demonstrated that regorafenib exhibits indications of clinical activity in multiple solid tumors, along with manageable toxicities. ${ }^{8}$

Because of the spectrum of target inhibition by regorafenib, promising preclinical data, and the clear unmet medical need in patients with GIST after failure of imatinib and sunitinib, we performed an investigator-initiated multicenter phase II trial of regorafenib in patients with advanced GIST after failure of at least imatinib and sunitinib. The primary objective of the trial was to assess clinical benefit as defined by the composite of complete response (CR), partial response (PR), and stable disease $(\mathrm{SD}) \geq 16$ weeks per RECIST 1.1. ${ }^{9}$ Secondary end points included assessment of PFS and evaluation of the safety and tolerability of regorafenib in this patient population. The protocol was reviewed and approved by the institutional review board at all participating sites, and the study was conducted in accordance with the ethical principles originating from the Declaration of Helsinki and Good Clinical Practice. All patients provided written informed consent before enrollment onto the study.

\section{PATIENTS AND METHODS}

\section{Patients}

Eligibility criteria included age $\geq 18$ years, histologically confirmed metastatic and/or unresectable GIST with progression while receiving or intolerance to imatinib and prior failure of sunitinib because of disease progression, Eastern Cooperative Oncology Group performance status of 0 or 1, and measurable disease per RECIST 1.1. ${ }^{9}$ Other eligibility factors included adequate organ and bone marrow function as defined by hemoglobin $\geq 9.0 \mathrm{~g} / \mathrm{dL}$, absolute neutrophil count $\geq 1,500 />\mathrm{L}$, platelets $\geq 100,000 / \mu \mathrm{L}$, international normalized ratio (or prothrombin time) and partial thromboplastin time $\leq 1.5 \times$ upper limit of normal (ULN); ALT and AST $\leq 2.5 \times$ ULN ( $\leq 5.0 \times$ ULN with liver metastases), alkaline phosphatase $<2.5 \times$ ULN $(\leq 5.0 \times$ ULN with liver metastases), total serum bilirubin $\leq 1.5 \times$ ULN, serum creatinine $\leq 1.5 \times$ ULN, serum sodium within normal limits, and glomerular filtration rate $\geq 30 \mathrm{~mL} / \mathrm{min} / 1.73 \mathrm{~m}^{2}$. Any number of previous therapies for GIST were permitted; however, prior exposure to sorafenib was not allowed. Other exclusion factors included major surgery or significant traumatic injury within 4 weeks before study entry; symptomatic or uncontrolled brain or CNS metastases; a history of clinically significant cardiac disease, congestive heart failure, unstable angina, or new-onset angina within the last 3 months; myocardial infarction within the past 6 months; uncontrolled hypertension as defined by systolic blood pressure $>140$ or diastolic blood pressure $>90$ despite optimal medical management; arterial or venous thrombotic or embolic events such as cerebrovascular accident (including transient ischemic attacks), deep vein thrombosis, or pulmonary embolism within the past 6 months; any major hemorrhage or bleeding event $>$ grade 3 within 4 weeks of start of study drug; renal failure requiring hemo- or peritoneal dialysis; and proteinuria $\geq$ grade 3 ( $>3.5$ g per 24 hours), measured by urine protein-to-creatinine ratio on a random urine sample.

\section{Study Design and Treatment}

This investigator-initiated trial was designed and written by two academic authors (S.G., G.D.D.). The investigational new drug regulatory permit under which this trial was conducted is held by the overall principal investigator (S.G.); monitoring of all sites was performed by the clinical trials office at the Dana-Farber/Harvard Cancer Center (Boston, MA).

Once enrolled, patients received regorafenib $160 \mathrm{mg}$ orally once daily on days 1 to 21 of a planned 28-day cycle (21 days on, 7 days off). Patients were able to continue study participation and regorafenib dosing unless one of the following criteria was met: patient decision to withdraw, unacceptable toxicity, or disease progression per RECIST 1.1 (unless in the opinion of the treating physician, the patient was exhibiting continued benefit from study drug, in which case the patient was allowed to remain on study at the discretion of the treating physician after approval by the overall principal investigator).

\section{Response Assessment and Toxicity}

Tumor assessments by standard anatomic imaging modalities (computed tomography or magnetic resonance imaging used consistently from baseline onward) were performed at the end of regorafenib dosing (day 21) after every two cycles. Response was determined per RECIST 1.1. Tolerability and adverse events were assessed continuously. Safety assessments included history and physical examination, measurement of blood pressure, complete blood count, serum chemistries, urinalysis, and measurement of urine protein. Toxicities were assessed per Common Terminology Criteria for Adverse Events 4.0. Criteria for dose modification and subsequent dose re-escalation are summarized in Appendix Tables A1 through A8 (online only).

\section{Correlative Studies}

When available, tumor tissue was analyzed for mutations in KIT and PDGFR- $\alpha$ genes. Tumors with no identifiable mutation in either KIT or PDGFR- $\alpha$ were genotyped for BRAF mutation. Patients who consented to optional tumor biopsies underwent tumor biopsy before the first dose of study drug, and a second biopsy was performed between days 10 and 21 of cycle one. Cell lysate protein preparations from the snap-frozen biopsies, protein quantification, electrophoresis, and immunoblotting were carried out as described previously. ${ }^{10}$ Chemiluminescence signals were captured and quantified using a Fujifilm LAS1000plus system (Fujifilm Medical Systems, Stamford, CT). Profiling of relevant signal transduction pathways was performed using antibodies to phospho-KIT Y703 (product No. 3073), phospho-AKT S473 (No. 9271), total AKT (No. 9272), phospho-MAPK p42/44 T202/Y204 (No. 9101), and total MAPK (No. 4695), all from Cell Signaling (Beverly, MA), and with phosphatidylinositol 3-kinase p85 from Millipore (Billerica, MA) and KIT antibody from DAKO (Carpinteria, CA).

\section{Statistical Analysis}

The primary efficacy end point was clinical benefit, defined as the proportion of patients with a confirmed objective response per RECIST 1.1 or $\mathrm{SD} \geq 16$ weeks. A one-stage design with 34 patients (31 eligible patients) was used to distinguish a favorable true clinical benefit rate (CBR) of $28 \%$ from a null rate of $10 \%$ with $90 \%$ power and $8 \%$ type I error. PFS was defined as the duration of time from date of registration to date of objective disease progression or death resulting from any cause, whichever occurred first. Overall survival (OS) was defined as the duration of time from date of registration to date of death.

All enrolled patients who received at least one dose of study drug were eligible for efficacy and toxicity analyses. Patients who were alive without documented disease progression were censored at the last disease assessment date. Five patients developed disease progression per RECIST 1.1 but remained on study because of continuing benefit per investigator decision. These five patients were classified as having disease progression at the time of RECIST-defined progressive disease for the efficacy analysis; however, they continued to be assessed for safety until removed from the protocol.

Descriptive statistics were used to characterize patients at study entry. Ninety-five percent two-sided exact binomial CIs were computed for CBR. PFS and OS were estimated using the Kaplan-Meier method. Pairwise comparisons of the differences in CBR and PFS by genotype were assessed by Fisher's exact and log-rank tests, respectively. A Bonferroni-adjusted $P$ value of $.017(.05 / 3)$ was considered statistically significant for all pairwise genotype comparisons. The data presented represent the study database as of July 28 , 2011. All analyses were conducted with SAS 9.2 (SAS Institute, Cary, NC).

\section{RESULTS}

Between February and December 2010, 34 patients with metastatic GIST were enrolled. One participant was judged ineligible for study drug dosing after enrollment, and therefore, 33 patients were eligible 


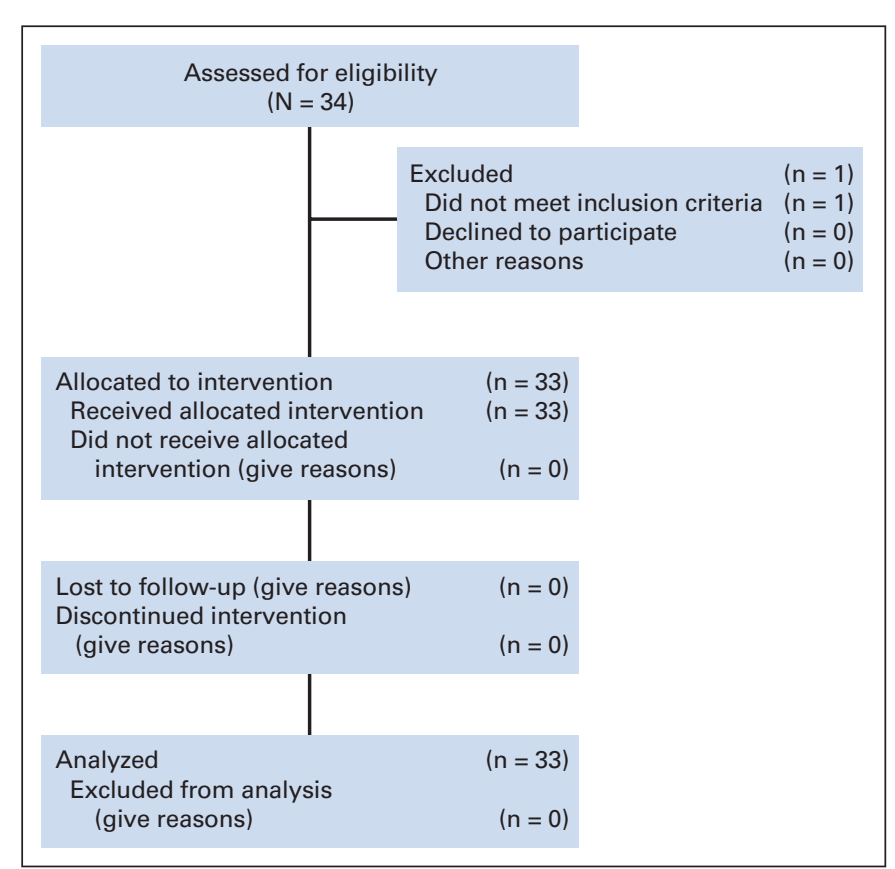

Fig 1. CONSORT diagram.

and received at least one dose of regorafenib (Fig 1). Baseline patient characteristics are listed in Table 1.

\section{Patient Disposition}

After a median follow-up of 10.9 months among living patients, 21 patients continued to receive regorafenib, 16 of whom remained progression free. Five patients continued to receive regorafenib after RECIST-defined disease progression because of investigator-assessed continued benefit. Continued benefit after disease progression included one patient with resection of a new solitary metastasis and four patients with either continued improvement in disease-related symptoms or slowing of the rate of tumor growth. These patients were categorized as having progressive disease at the time of progression and continued to be observed for toxicity. Twelve patients discontinued study drug: six for RECIST-defined disease progression, three for investigator-assessed clinical progression, one for an unrelated intercurrent illness, one per patient choice (because of hardship of travel to a study site), and one per investigator decision after an adverse event (large intratumoral bleed leading to prolonged ileus in cycle two, an event felt to be possibly related to study drug, perhaps resulting from rapid tumor necrosis in response to regorafenib).

\section{Toxicity}

As of July 28, 2011, 280 cycles of regorafenib had been administered, with a median of eight cycles administered per patient (range, two to 17 cycles). Toxicities of any grade occurring in at least $25 \%$ of patients are shown in Figure 2. The most commonly observed toxicities of any grade were hand-foot-skin reaction, fatigue, hypertension, and diarrhea occurring in $85 \%, 79 \%, 67 \%$, and $61 \%$ of patients, respectively. A majority of these were grade 1 or 2 (Fig 2).

Grade 3 toxicities occurring in $\geq 5 \%$ of patients and all grade 4 toxicities are listed in Table 2; these included hypertension, hand-footskin reaction, and hypophosphatemia (with no clinical sequelae) in $36 \%, 24 \%$ and $15 \%$, of patients, respectively.

\begin{tabular}{|c|c|c|}
\hline Characteristic & No. & $\%$ \\
\hline \multicolumn{3}{|l|}{ Age, years } \\
\hline Median & \multicolumn{2}{|c|}{56} \\
\hline Range & \multicolumn{2}{|c|}{$25-76$} \\
\hline \multicolumn{3}{|l|}{ Sex } \\
\hline Female & 14 & 42 \\
\hline Male & 19 & 58 \\
\hline \multicolumn{3}{|l|}{ ECOG PS } \\
\hline 0 & 23 & 70 \\
\hline 1 & 10 & 30 \\
\hline \multicolumn{3}{|l|}{ No. of prior regimens } \\
\hline Median & \multicolumn{2}{|c|}{2} \\
\hline Range & \multicolumn{2}{|c|}{$2-10$} \\
\hline \multicolumn{3}{|c|}{ Time receiving first-line imatinib, months } \\
\hline Median & \multicolumn{2}{|c|}{21} \\
\hline Range & \multicolumn{2}{|c|}{$1-79$} \\
\hline \multicolumn{3}{|l|}{ Reason for imatinib failure } \\
\hline Progression of disease & 30 & 90 \\
\hline Intolerance & 3 & 10 \\
\hline \multicolumn{3}{|l|}{ Time receiving sunitinib, months } \\
\hline Median & \multicolumn{2}{|c|}{13} \\
\hline Range & \multicolumn{2}{|c|}{$2-55$} \\
\hline \multicolumn{3}{|l|}{ Primary kinase mutation* } \\
\hline KIT exon 11 & \multicolumn{2}{|l|}{19} \\
\hline KIT exon 9 & \multicolumn{2}{|l|}{3} \\
\hline WT for KIT and PDGFR- $\alpha \dagger$ & \multicolumn{2}{|l|}{8} \\
\hline BRAF exon 15 & \multicolumn{2}{|l|}{1} \\
\hline \multicolumn{3}{|c|}{$\begin{array}{l}\text { NOTE. Time receiving prior therapy may not correlate with time to } \\
\text { RECIST progression. } \\
\text { Abbreviations: ECOG PS, Eastern Cooperative Oncology Group performance } \\
\text { status; GIST, GI stromal tumor; WT, wild type. } \\
\text { "Available for } 30 \text { patients; three patients had insufficient material for analysis. } \\
\text { tEight patients (WT) had GIST with no mutations in KIT exons } 9,11,13 \text {, or } \\
17 \text { or PDGFR- } \alpha \text { exons } 12,14 \text {, or } 18 \text {. Two of these eight patients were also } \\
\text { tested for KIT exons } 14,15,1,6 \text { and } 18 \text { and found to be WT; all WT patients } \\
\text { were then screened for mutations in BRAF exon } 15 \text {. }\end{array}$} \\
\hline
\end{tabular}

Twenty-seven $(82 \%)$ of 33 patients required dose reduction at some point on study based on protocol-defined toxicity. The most common reasons for dose reduction were hypertension and handfoot-skin reaction. Of the 27 patients with dose reduction, 12 patients were dose reduced to $120 \mathrm{mg}, 11$ patients to $80 \mathrm{mg}$, and four patients to $40 \mathrm{mg}$ per day. Of the 15 patients who were dose reduced below 120 mg per day, 11 tolerated subsequent re-escalation of regorafenib dose from 120 to $160 \mathrm{mg}$ per day, including one patient who had been reduced to $40 \mathrm{mg}$ per day. In total, 11 patients tolerated a final dose of $160 \mathrm{mg}$ per day. An additional 12 patients tolerated a final dose of 120 mg per day.

\section{Efficacy}

Clinical benefit (CR, PR, and SD $\geq 16$ weeks) was documented in 26 (75\%) of 33 patients (95\% CI, 61\% to 91\%), comprising four PRs and 22 instances of SD. Four patients had SD for $\leq 16$ weeks, two patients demonstrated disease progression at first tumor evaluation, and one patient was not evaluable for response because of withdrawal from study before first disease assessment.

The Kaplan-Meier estimate of PFS for the entire cohort is shown in Figure 3A. Median PFS is 10.0 months (95\% CI, 8.3 to 14.9 months). Actuarial PFS is summarized in Table 3. 


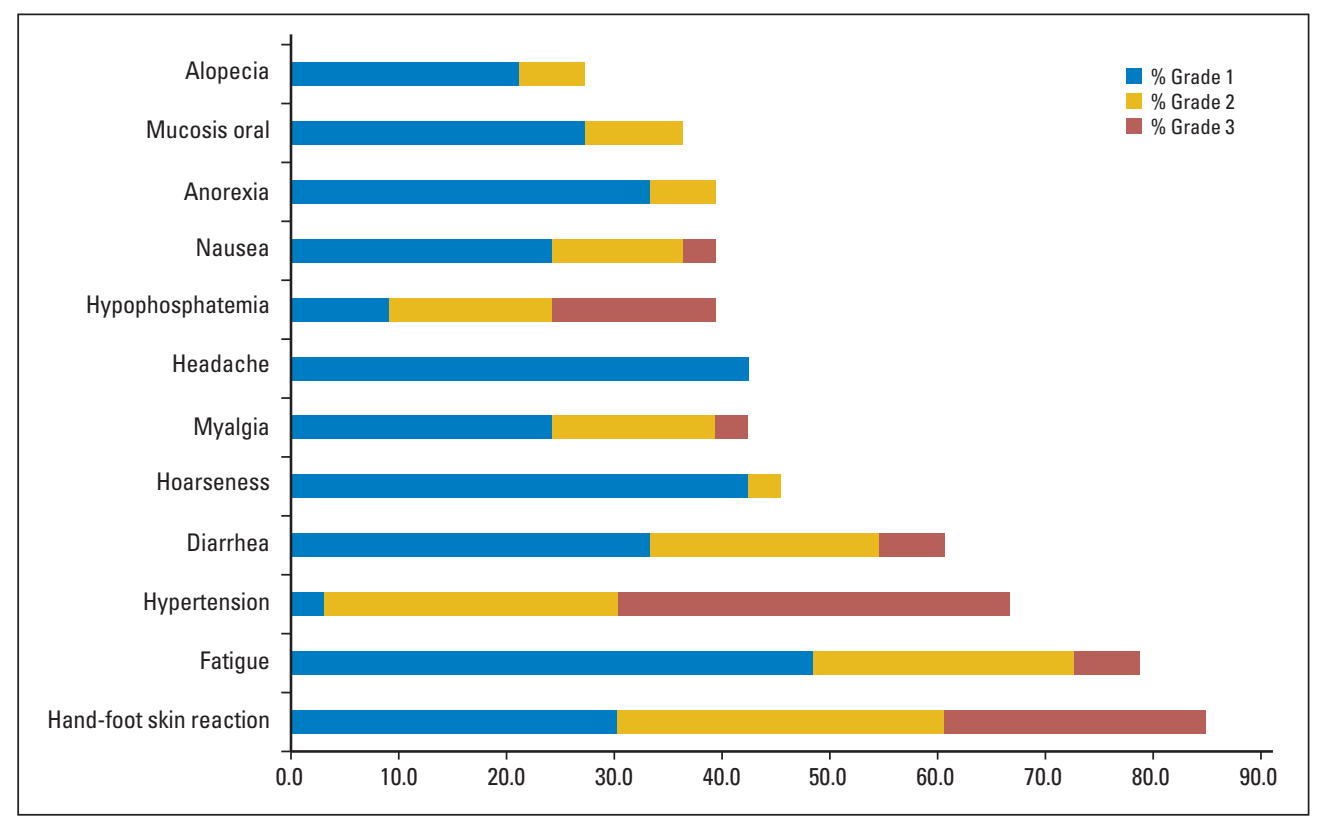

Fig 2. Toxicities of any grade, at least possibly study drug related, occurring in $>$ $25 \%$ of patients.
There were six patient deaths, five secondary to disease progression and one secondary to an unrelated intercurrent illness (complications after replacement of prosthetic aortic valve). Median OS has not yet been reached (Figs $3 \mathrm{~A}$ to $3 \mathrm{C}$ ).

\section{Correlative Science Studies}

Primary tumor genotype was available for 30 patients, as shown in Table 1; there was no PDGFR- $\alpha$-mutant GIST in this trial. The end point of protocol-defined clinical benefit was documented in patients with tumors harboring mutations in KIT exons 9 and 11 as well as for GIST in which no KIT or PDGRF- $\alpha$ mutations were detected (ie, wild-type GIST). There was no statistically significant difference in the rate of clinical benefit among genotype groups (data provided in Appendix Table A9, online only), although these comparative subsets were small. The Kaplan-Meier estimate of PFS by tumor genotype is shown in Figure 3B. After pairwise comparisons, patients with tumors with primary exon 11 KIT mutations had a significantly longer PFS when compared with patients with tumors with primary exon 9 KIT

Table 2. Grade 3 Toxicities Occurring in $>5 \%$ of Patients and All Grade 4 Toxicities*

\begin{tabular}{|c|c|c|c|c|}
\hline \multirow[b]{2}{*}{ Toxicity } & \multicolumn{2}{|c|}{ Grade 3} & \multicolumn{2}{|c|}{ Grade 4} \\
\hline & No. & $\%$ & No. & $\%$ \\
\hline Hypertension & 12 & 36 & 0 & 0 \\
\hline Hand-foot-skin reaction & 8 & 24 & 0 & 0 \\
\hline Hypophosphatemiat & 5 & 15 & 0 & 0 \\
\hline Rash & 3 & 9 & 0 & 0 \\
\hline Fatigue & 2 & 6 & 0 & 0 \\
\hline Diarrhea & 2 & 6 & 0 & 0 \\
\hline Lipase elevationt & 2 & 6 & 0 & 0 \\
\hline Hyperuricemia & 0 & 0 & 2 & 6 \\
\hline Thrombotic event & 0 & 0 & 1 & 3 \\
\hline
\end{tabular}

*At least possibly study drug related.

†All elevations in lipase and decreases in phosphate were asymptomatic. mutations $(P=.01)$, although it should be noted that there were only three patients with exon 9-mutant tumors. There was no significant difference in PFS when comparing patients with wild-type GIST with patients with either exon 9 or 11 KIT-mutant tumors (data provided in Appendix Table A10, online only). The one patient with GIST containing BRAF exon 15 mutation exhibited rapid disease progression despite regorafenib administration.

\section{Serial Tumor Biopsy Analyses of Signal Transduction Pathways}

Immunoblotting of matched serial GIST biopsies, taken at baseline and then during the second week of regorafenib dosing, demonstrated target inhibition, with decreased levels of KIT phosphorylation as well as variable inhibition of AKT and MAPK phosphorylation in four patients with imatinib- and sunitinib-resistant KIT activation loop mutations D820Y and N822K (Fig 4; Appendix Fig A1, online only). All four patients achieved protocol-defined clinical benefit. Two of the four patients remain on study without disease progression (patient case No. 9 for $>11$ months and patient case No. 21 for $>7$ months). One patient developed disease progression at 5.7 months (patient case No. 15); another was censored for response assessment after undergoing surgical removal of all residual disease after approximately 7 months (patient case No. 19) and continues to receive study drug. Biopsies at the time of disease progression while receiving regorafenib have not yet been analyzed; however, this is planned in the trial.

\section{DISCUSSION}

GIST is a disease driven by aberrant signaling through constitutive activation of KIT or PDGFR- $\alpha$ in the majority of tumors. Imatinib is a selective inhibitor of KIT and PDGFR- $\alpha$, which leads to excellent initial disease control for most patients with metastatic disease. Resistance to imatinib appears within a median of 2 years, and after 10 


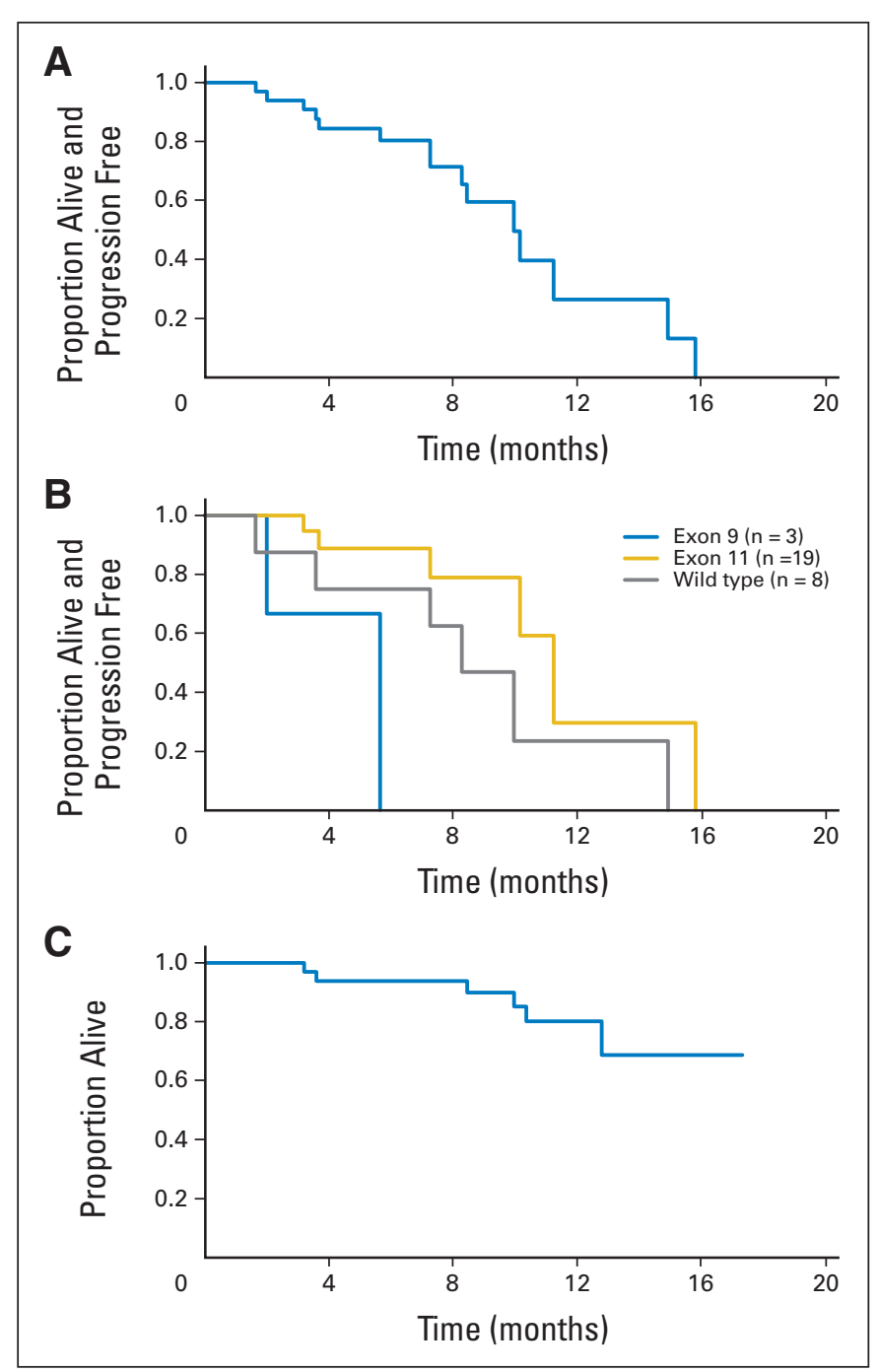

Fig 3. Kaplan-Meier estimates of (A) progression-free survival (PFS) for the entire cohort ( $n=33$ ); (B) PFS by genotype for patients with exon 9 KIT mutations $(\mathrm{n}=3)$, exon 11 KIT mutations ( $\mathrm{n}=19$ ), and wild type for both KIT and PDGFRA $(n=8)$; and $(C)$ overall survival (OS) for the entire cohort $(n=33)$. At time of analysis, 16 patients remained without disease progression. Median PFS for the entire cohort was 10.0 months $(95 \% \mathrm{Cl}, 8.3$ to 14.9 months); median OS has not yet been reached.

years, more than $80 \%$ of patients will ultimately develop resistance. ${ }^{11}$ Sunitinib, a multitargeted TKI, has more potent inhibitory activity against the KIT kinase and has proven benefit in patients with imatinib-resistant GIST; however, after second-line sunitinib therapy, GIST subclones resistant to both sunitinib and imatinib generally appear within 6 to 12 months. There are no approved third-line

\begin{tabular}{|ccr|}
\hline \multicolumn{3}{|c|}{ Table 3. Actuarial Progression-Free Rate for the Entire Cohort } \\
\hline Months & Progression-Free Rate (\%) & $95 \% \mathrm{Cl}$ \\
\hline 4 & 84 & 66 to 93 \\
6 & 80 & 61 to 91 \\
8 & 72 & 50 to 85 \\
10 & 50 & 24 to 71 \\
\hline
\end{tabular}

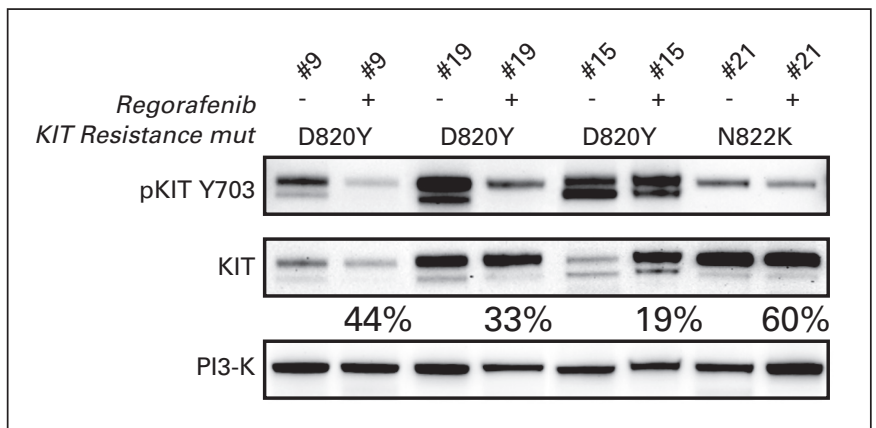

Fig 4. Immunoblotting analyses of matched pre- and postregorafenib GI stromal tumor biopsies from four patients demonstrate KIT inactivation, expressed as percentage of preregorafenib KIT activation remaining in the matched postregorafenib sample [(postregorafenib phospho-KIT/total-KIT) $\div$ (preregorafenib phospho-KIT/total KIT)]. Genomic analyses demonstrated KIT activation loop imatinib- and sunitinib-resistance mutations (mut) in each biopsy (D820Y in three patients, N822K in one patient). Total phosphatidylinositol 3-kinase (PI3-K) stain is a well-validated loading control, which serves as an indicator of cellular protein content in each biopsy.

therapies for this patient population. Previous phase II studies, primarily reported as retrospective series or preliminary reports, have evaluated other kinase inhibitors in patients with advanced GIST resistant to imatinib and sunitinib. ${ }^{12-18}$ For example, preliminary data from two independent phase II trials using sorafenib in this patient population have demonstrated a median PFS of approximately 5 months. ${ }^{14,15}$ A recently reported phase II trial and preliminary report of a phase III trial of nilotinib in this population demonstrated a median PFS of 118 days and 109 days, respectively. ${ }^{12,13}$ Additional studies of other agents have similarly demonstrated modest response rates and median PFS in the range of 2 to 5 months, ${ }^{17,18}$ although direct comparisons with prior series cannot be made.

Our current study demonstrates that regorafenib has notable anticancer activity in patients with advanced GIST after resistance to both imatinib and sunitinib. Secondary mutations in KIT account for the majority of TKI resistance. ${ }^{19-22}$ These mutations cluster in the exons encoding the ATP (and drug) binding pocket (exons 13 and 14) and in the exons encoding the kinase activation loop (exons 17 and 18). ${ }^{19,20}$ In addition, it has been shown that different secondary KIT mutations can be found in different tumors of any single patient, highlighting the biologic complexity of TKI-resistant GIST. ${ }^{20}$ On the basis of preclinical studies, both imatinib and sunitinib are ineffective against mutations affecting the activation loop of either KIT or PDGFR- $\alpha{ }^{19}$ Therefore, one potential explanation for the benefit of regorafenib after failure of both imatinib and sunitinib could be a structurally unique interaction and inhibitory activity against these activation loop kinase mutations. Analysis of serial GIST biopsies in this trial demonstrated decreased phosphorylation of KIT and downstream signaling proteins (including AKT as well as MAPK) after study drug dosing, confirming KIT inhibition by regorafenib in these patients. It is also possible that regorafenib inhibits other signaling pathways (such as fibroblast growth factor receptor 1), which may contribute to GIST resistance through heretofore unrecognized compensatory signaling pathways.

The observed toxicity of regorafenib in this trial was similar to that reported in previous phase I trials with this agent, with hand-footskin reaction, hypertension, fatigue, and diarrhea the most common adverse events observed. ${ }^{8,23,24}$ These toxicities are also consistent with 
the toxicity profile of other kinase inhibitors with a similar target spectrum. Despite the majority of patients requiring at least one dose reduction for toxicity, many patients were able to have the regorafenib dose re-escalated without recurrence of unacceptable adverse effects. Approximately one third of patients could tolerate a maximum dose $\leq 80 \mathrm{mg}$ per day. Because patients were able to re-escalate the dose, we were not able to assess any possible dose-response relationships in this study. Only one patient discontinued participation in the study because of toxicity, suggesting that in this cohort, toxicity was manageable with dose modification and other supportive measures.

In summary, regorafenib is a novel orally available multikinase inhibitor with notable activity in patients with advanced GIST after objective failure of both prior imatinib and sunitinib. The median PFS $>10$ months observed in these heavily pretreated patients supports the hypothesis that regorafenib may be a uniquely active agent in the management of GIST after treatment with imatinib and sunitinib. An international phase III trial evaluating regorafenib in this population is under way to provide a definitive evaluation of this question.

\section{AUTHORS' DISCLOSURES OF POTENTIAL CONFLICTS} OF INTEREST

Although all authors completed the disclosure declaration, the following author(s) indicated a financial or other interest that is relevant to the subject matter under consideration in this article. Certain relationships marked with a " $U$ " are those for which no compensation was received; those relationships marked with a "C" were compensated. For a detailed description of the disclosure categories, or for more information about ASCO's conflict of interest policy, please refer to the Author Disclosure Declaration and the Disclosures of Potential Conflicts of Interest section in Information for Contributors.

Employment or Leadership Position: None Consultant or Advisory Role: Suzanne George, Novartis (C), Pfizer (C); Michael C. Heinrich, MolecularMD (C), Novartis (C), Pfizer (C); Andrew J. Wagner, Novartis
(C), Pfizer (C); Edwin Choy, Amgen (C), sanofi-aventis (C); George D. Demetri, Eli Lilly/ImClone (C), Genentech (C), GlaxoSmithKline (C), Infinity Pharmaceuticals (C), Kolltan Pharmaceuticals (C), Novartis (C), Pfizer (C) Stock Ownership: Michael C. Heinrich, MolecularMD; George D. Demetri, Kolltan Pharmaceuticals Honoraria: Michael C. Heinrich, Novartis; George D. Demetri, Novartis, Pfizer Research Funding: Suzanne George, Bayer, Novartis, Pfizer; Michael C. Heinrich, ARIAD Pharmaceuticals, AROG Pharmaceuticals, ImClone, Novartis; Edwin Choy, AstraZeneca, Novartis; Jeffrey T. Yap, Bayer, Bristol-Myers Squibb, Toshiba; Annick D. Van den Abbeele, Bayer; Jonathan A. Fletcher, Bayer; George D. Demetri, Bayer, Eli Lilly/ImClone, Infinity Pharmaceuticals, Novartis, Pfizer Expert Testimony: Edwin Choy, Martin, Magnuson, McCarthy, and Kenny (C), Michaud, Mittlemark, Marowitz, and Asrani (C), Simons, Perrine, Mayer, and Bergman (C); George D. Demetri, Bayer (U), Infinity Pharmaceuticals (U) Other Remuneration: None

\section{AUTHOR CONTRIBUTIONS}

Conception and design: Suzanne George, Qian Wang, Jeffrey T. Yap, Annick D. Van den Abbeele, Judith B. Manola, Sarah M. Solomon, George D. Demetri

Administrative support: Suzanne George

Provision of study materials or patients: Suzanne George, Michael C. Heinrich, James E. Butrynski, Jeffrey A. Morgan, Andrew J. Wagner, Edwin Choy, Margaret von Mehren

Collection and assembly of data: Suzanne George, Michael C. Heinrich, Christopher L. Corless, James E. Butrynski, Jeffrey A. Morgan, Andrew J. Wagner, Edwin Choy, William D. Tap, Jeffrey T. Yap, Annick D. Van den Abbeele, Jonathan A. Fletcher, Margaret von Mehren,

George D. Demetri

Data analysis and interpretation: Suzanne George, Qian Wang, Michael C. Heinrich, Christopher L. Corless, Meijun Zhu, Andrew J. Wagner, Jeffrey T. Yap, Annick D. Van den Abbeele, Judith B. Manola, Jonathan A. Fletcher, Margaret von Mehren, George D. Demetri

Manuscript writing: All authors

Final approval of manuscript: All authors

\section{REFERENCES}

1. Hirota S, Isozaki K, Moriyama Y, et al: Gainof-function mutations of c-kit in human gastrointestinal stromal tumors. Science 279:577-580, 1998

2. Joensuu H, Roberts PJ, Sarlomo-Rikala $M$, et al: Effect of the tyrosine kinase inhibitor STI571 in a patient with a metastatic gastrointestinal stromal tumor. N Engl J Med 344:1052-1056, 2001

3. Demetri GD, von Mehren M, Blanke CD, et al: Efficacy and safety of imatinib mesylate in advanced gastrointestinal stromal tumors. N Engl J Med 347: 472-480, 2002

4. Demetri GD, van Oosterom AT, Garrett CR, et al: Efficacy and safety of sunitinib in patients with advanced gastrointestinal stromal tumour after failure of imatinib: A randomised controlled trial. Lancet 368:1329-1338, 2006

5. Blanke CD, Demetri GD, von Mehren M, et al: Long-term results from a randomized phase II trial of standard- versus higher-dose imatinib mesylate for patients with unresectable or metastatic gastrointestinal stromal tumors expressing KIT. J Clin Oncol 26:620-625, 2008

6. Gramza AW, Corless CL, Heinrich MC: Resistance to tyrosine kinase inhibitors in gastrointestinal stromal tumors. Clin Cancer Res 15:7510-7518, 2009
7. Wilhelm SM, Dumas J, Adnane $L$, et al: Regorafenib (BAY 73-4506): A new oral multikinase inhibitor of angiogenic, stromal and oncogenic receptor tyrosine kinases with potent preclinical antitumor activity. Int J Cancer 129:245-255, 2011

8. Frost $A$, Buechert $M$, Unger $C$, et al: Phase I study of BAY 73-4506, an inhibitor of oncogenic and angiogenic kinases, in patients with advanced solid tumors: Final results of a dose-escalation study. J Clin Oncol 26:126s, 2008 (suppl; abstr 2558)

9. Eisenhauer EA, Therasse P, Bogaerts J, et al: New response evaluation criteria in solid tumours: Revised RECIST guideline (version 1.1). Eur J Cancer 45:228-247, 2009

10. Duensing A, Medeiros F, McConarty B, et al: Mechanisms of oncogenic KIT signal transduction in primary gastrointestinal stromal tumors (GISTs). Oncogene 23:3999-4006, 2004

11. von Mehren $M$, Heinrich $M C$, Joensuu $H$, et al: Follow-up results after 9 years (yrs) of the ongoing, phase II B2222 trial of imatinib mesylate (IM) in patients (pts) with metastatic or unresectable KIT+ gastrointestinal stromal tumors (GIST). J Clin Oncol 29:609s, 2011 (suppl; abstr 10016)

12. Sawaki $A$, Nishida $T$, Doi $T$, et al: Phase 2 study of nilotinib as third-line therapy for patients with gastrointestinal stromal tumor. Cancer [epub ahead of print on March 31, 2011]

13. Reichardt $P$, Blay J, Gelderblom $H$, et al: Phase III trial of nilotinib in patients with advanced gastrointestinal stromal tumor (GIST): First results from ENEST g3. J Clin Oncol 28:702s, 2010 (suppl; abstr 10017)

14. Kindler HL, Campbell NP, Wroblewski K, et al: Sorafenib (SOR) in patients (pts) with imatinib (IM) and sunitinib (SU)-resistant (RES) gastrointestinal stromal tumors (GIST): Final results of a University of Chicago Phase II Consortium trial. J Clin Oncol 29:607s, 2011 (suppl; abstr 10009)

15. Ryu M, Park SH, Ryoo B, et al: A phase II study of sorafenib in patients with metastatic or unresectable gastrointestinal stromal tumors with failure of both imatinib and sunitinib: A KGSG study. J Clin Oncol 29:607s, 2011 (suppl; abstr 10010)

16. Trent JC, Wathen K, von Mehren M, et al: A phase II study of dasatinib for patients with imatinib-resistant gastrointestinal stromal tumor (GIST). J Clin Oncol 29:606s, 2011 (suppl; abstr 10006)

17. Schöffski $P$, Reichardt $P$, Blay JY, et al: $A$ phase I-II study of everolimus (RAD001) in combination with imatinib in patients with imatinib-resistant gastrointestinal stromal tumors. Ann Oncol 21:19901998, 2010

18. Joensuu $H$, De Braud F, Grignagni $G$, et al: Vatalanib for metastatic gastrointestinal stromal tumour (GIST) resistant to imatinib: Final results of a phase II study. Br J Cancer 104:1686-1690, 2011 
19. Heinrich M, Corless $C$, Blanke C, et al: Molecular correlates of imatinib resistance in gastrointes tinal stromal tumors. J Clin Oncol 24:4764-4774, 2006

20. Liegl B, Kepten I, Le C, et al: Heterogeneity of kinase inhibitor resistance mechanisms in GIST. J Pathol 216:64-74, 2008

21. Heinrich $M C$, Maki RG, Corless $C L$, et al: Primary and secondary kinase genotypes correlate with the biological and clinical activity of sunitinib in imatinib resistant gastrointestinal stromal tumor. J Clin Oncol 26:5352-5359, 2008

22. Wang WL, Conley A, Reynoso D, et al: Mechanisms of resistance to imatinib and sunitinib in gastrointestinal stromal tumor. Cancer Chemother Pharmacol 67:S15-S24, 2011 (suppl 1)

23. Eisen $T$, Joensuu $H$, Nathan $P$, et al: Phase II trial of the oral multikinase inhibitor BAY 73-4506 as 1st-line therapy in patients with metastatic or unresectable renal cell cancer (RCC). Eur J Cancer 7:424, 2009 (suppl)

24. Strumberg D, Scheulen ME, Frost $A$, et al: Phase I study of BAY 73-4506, an inhibitor of oncogenic and angiogenic kinases, in patients with advanced refractory colorectal carcinoma (CRC). J Clin Oncol 27:161s, 2009 (suppl; abstr 3560)

\section{Every 5 Minutes Research Published in JCO Is Cited in Other Peer-Reviewed Journals}

As reported by Thomson Reuters in its 2010 Journal Citation Reports ${ }^{\circledR}$, Journal of Clinical Oncology's Impact Factor has increased to 18.970 from 17.793. This is JCO's sixth straight year-on-year increase.

In number of citations, JCO ranks second among oncology journals and ranks 17th among all 8,005 scientific journals surveyed. JCO articles were cited more than 114,000 times in 2010.

JCO has published so much research-changing and practice-changing science over the years that, in 2010, a JCO article was cited every 5 minutes, on average, in another peer-reviewed journal.

If you want to have your research read by the largest, most discerning international audience, you need to publish in JCO. And if you want to read the most important research in clinical oncology, you need to subscribe to JCO.

To submit a manuscript, visit submit.jco.org.

To subscribe or activate, visit jco.org/subscriptions. 